\title{
A Study on Some Multi-Valued Interpolative Contractions
}

\author{
Seher Sultan Yeşilkaya ${ }^{*}$, Cafer Aydın² and Yaşar Aslan ${ }^{3}$
}

\begin{abstract}
In the present study, we introduce a new approach to interpolative mappings in fixed point theory by combining the ideas of Nadler [1], Karapınar et. al.[2, 3], Jleli and Samet [4]. We introduce some fixed point theorems for interpolative single and multi-valued Kannan type and Reich Rus Ćirić type $\theta$-contractive mappings on complete metric spaces and prove some fixed point results for these mappings. These results extend the main results of many comparable results from the current literature. Also, we give an example to show that our main theorems are applicable.
\end{abstract}

Keywords: Fixed point, Metric spaces, Multi-valued contractive mapping 2010 AMS: Primary 47H10, Secondary 54H25

1 Institute of Science and Technology, Kahramanmaraş Sütçü Imam University, Kahramanmaraş, 46040, Turkey ORCID: 0000-0002-1748-2398 ${ }^{2}$ Department of Mathematics, Kahramanmaraş Sütçü Imam University, Kahramanmaraş, 46040, Turkey ORCID: 0000-0002-3707-5837 ${ }^{3}$ Department of Mathematics, Kahramanmaraş Sütçü Imam University, Kahramanmaraş, 46040, Turkey ORCID: 0000-0002-8827-2128 *Corresponding author: sultanseher20@gmail.com Received: 12 September 2020, Accepted: 15 December 2020, Available online: 22 December 2020

\section{Introduction}

Banach [5] introduced a famous fundamental fixed point theorem, also known as the Banach contraction principle. There are various extensions and generalizations of the Banach contraction principle in the literature see for example Kannan's [6], Reich [7] and see also Ćirić's [8].

In 1968, Kannan [6] proved a new fixed point theorem and considered the following contractive type:

$$
d(F \eta, F \omega) \leq \lambda[d(\eta, F \eta)+d(\omega, F \omega)]
$$

where $\lambda \in\left[0, \frac{1}{2}\right)$. In [2], the notion of an interpolation Kannan type contractive was introduced. On the other hand, Reich, Rus and Ćirić $[7,9,10,11,12,13,14]$ combined and improved both Banach and Kannan fixed point theorems. Recently, Karapınar et. al., [3] proved interpolative Reich Rus Ćirić type contractive mappings on partial metric spaces.

In 1969, using Pompeiu-Hausdorff metric, Nadler [1] introduced the notion of multi-valued contraction mapping and proved a multi-valued version of the well known Banach contraction principle. Denote by $P(X)$ the family of all nonempty subsets of $X, C(X)$ the family of all nonempty, closed subsets of $X, C B(X)$ the family of all nonempty, closed and bounded subsets of $X$ and $K(X)$ the family of all nonempty compact subsets of $X$. It is clear that, $K(X) \subseteq C B(X) \subseteq C(X) \subseteq P(X)$. It is well known that, $H: C B(X) \times C B(X) \rightarrow \mathbb{R}$ is defined by, for every $F, G \in C B(X)$,

$$
H(F, G)=\max \left\{\sup _{f \in F} d(f, G), \sup _{g \in G} d(g, F)\right\}
$$


is a metric on $C B(X)$, which is called the Pompeiu-Hausdorff metric induced by $d$, where $D(f, G)=\inf \{d(f, g): g \in G\}$ and $D(F, G)=\sup \{D(f, G): f \in F\}$. Additionally, we will use the following lemma:

Lemma 1.1. Let $(X, d)$ metric spaces and $F$ compact subsets of $X$. Afterwards, for $x \in X$, there exist $f \in F$, such that

$$
d(x, f)=D(x, F) .
$$

Lemma 1.2. [1] Let $F$ and $G$ be nonempty closed and bounded subsets of a metric space. Therefore, for any $f \in F$,

$$
D(f, G) \leq H(F, G) .
$$

Lately, Jleli and Samet [4] introduced a new type of contractions called $\theta$-contraction. They introduced the family of all functions, $\theta:(0, \infty) \rightarrow(1, \infty)$ supplying the following particulars by $\Theta$ :

$\left(\Theta_{1}\right) \theta$ is nondecreasing;

$\left(\Theta_{2}\right)$ For each sequence $\left\{s_{n}\right\} \subset(0, \infty), \lim _{n \rightarrow \infty} \theta\left(s_{n}\right)=1$ if and only if $\lim _{n \rightarrow \infty} s_{n}=0^{+}$;

$\left(\Theta_{3}\right)$ There exist $m \in(0,1)$ and $z \in(0, \infty]$ such that $\lim _{s \rightarrow 0^{+}} \frac{\theta(s)-1}{s^{m}}=z$.

In section 1, some basic definitions and theorem in the literature that will be used in the paper are given. In section 2, by using the approach of Nadler [1], Jleli and Samet [4] and Karapınar et. al.[2, 3], we introduce the notion of extended interpolative single and multi-valued Kannan type and Reich Rus Ćirić type $\theta$-contractive mappings.

\section{Main Results}

Firstly, let us start with the definition of interpolative Kannan type $\theta$-contractive mapping.

Definition 2.1. Let $(X, d)$ be a complete metric space and $\theta \in \Theta$. A mapping $F: X \rightarrow X$ is said to be an interpolative Kannan type $\theta$-contractive mapping if $\theta \in \Theta$ and there exist $\lambda \in[0,1), \alpha \in(0,1)$ such that

$$
\theta(d(F \eta, F \omega)) \leq[\theta(d(\eta, F \eta))]^{\lambda \alpha}[\theta(d(\omega, F \omega))]^{\lambda(1-\alpha)}
$$

for all $\eta, \omega \in X$.

Theorem 2.2. Let $(X, d)$ be a complete metric space and $F: X \rightarrow X$ be an interpolative Kannan type $\theta$-contractive, then $F$ has a fixed point in $X$.

Proof. Starting from $\eta_{0} \in X$, consider $\left\{\eta_{n}\right\}$ given as $\eta_{n}=F \eta_{n-1}$ for all positive integer $n$. If there is $n_{0}$ so that $\eta_{n 0}=\eta_{n 0+1}$ then $\eta_{n 0}$ is a fixed point of $F$. Assume that $\eta_{n} \neq \eta_{n+1}$ for all $n \geq 0$. Taking $\eta=\eta_{n-1}$ and $\omega=\eta_{n}$ in (2.1), one writes

$$
\theta\left(d\left(\eta_{n}, \eta_{n+1}\right)\right) \leq\left[\theta\left(d\left(\eta_{n-1}, \eta_{n}\right)\right)\right]^{\lambda \alpha}\left[\theta\left(d\left(\eta_{n}, \eta_{n+1}\right)\right)\right]^{\lambda(1-\alpha)} .
$$

If

$$
d\left(\eta_{n-1}, \eta_{n}\right)<d\left(\eta_{n}, \eta_{n+1}\right),
$$

then, from (2.2) we obtain

$$
\theta\left(d\left(\eta_{n}, \eta_{n+1}\right)\right) \leq\left[\theta\left(d\left(\eta_{n}, \eta_{n+1}\right)\right)\right]^{\lambda(1-\alpha+\alpha)}=\left[\theta\left(d\left(\eta_{n}, \eta_{n+1}\right)\right)\right]^{\lambda}
$$

which is a contradiction. Thus, for all $n \in \mathbb{N}$

$$
\theta\left(d\left(\eta_{n}, \eta_{n+1}\right)\right) \leq\left[\theta\left(d\left(\eta_{n-1}, \eta_{n}\right)\right)\right]^{\lambda} .
$$

Using (2.3) we have

$$
\theta\left(d\left(\eta_{n}, \eta_{n+1}\right)\right) \leq\left[\boldsymbol{\theta}\left(d\left(\eta_{n-1}, \eta_{n}\right)\right)\right]^{\lambda} \leq\left[\boldsymbol{\theta}\left(d\left(\eta_{n-2}, \eta_{n-1}\right)\right)\right]^{\lambda^{2}} \leq \cdots \leq\left[\boldsymbol{\theta}\left(d\left(\eta_{0}, \eta_{1}\right)\right)\right]^{\lambda^{n}} .
$$

Letting $n \rightarrow \infty$ in (2.4) we obtain

$$
\lim _{n \rightarrow \infty} \theta\left(d\left(\eta_{n}, \eta_{n+1}\right)\right)=1,
$$


From $\left(\Theta_{2}\right)$ we get

$$
\lim _{n \rightarrow \infty} d\left(\eta_{n}, \eta_{n+1}\right)=0^{+}
$$

and from $\left(\Theta_{3}\right)$, there exist $a \in(0,1)$ and $b \in(0, \infty]$ such that

$$
\lim _{n \rightarrow \infty} \frac{\theta\left(d\left(\eta_{n}, \eta_{n+1}\right)\right)-1}{\left(d\left(\eta_{n}, \eta_{n+1}\right)\right)^{a}}=b .
$$

Suppose that $b<\infty$. In this case, let $S=\frac{b}{2}>0$. Using the definition of the limit, there exist $n_{0} \in \mathbb{N}$ such that

$$
\left|\frac{\theta\left(d\left(\eta_{n}, \eta_{n+1}\right)\right)-1}{\left(d\left(\eta_{n}, \eta_{n+1}\right)\right)^{a}}-b\right| \leq S, \text { for all } n \geq n_{0} .
$$

This implies that

$$
\frac{\theta\left(d\left(\eta_{n}, \eta_{n+1}\right)\right)-1}{\left(d\left(\eta_{n}, \eta_{n+1}\right)\right)^{a}} \geq b-S=S, \text { for all } n \geq n_{0} .
$$

Then

$$
n\left(d\left(\eta_{n}, \eta_{n+1}\right)\right)^{a} \leq \operatorname{Rn}\left[\theta\left(d\left(\eta_{n}, \eta_{n+1}\right)\right)-1\right]
$$

for all $n \geq n_{0}$ where $R=\frac{1}{S}$. Now suppose that $b=\infty$ and $S>0$ be an arbitrary positive number. Using the definition the limit, there exist $n_{0} \in \mathbb{N}$ such that

$$
\frac{\theta\left(d\left(\eta_{n}, \eta_{n+1}\right)\right)-1}{\left(d\left(\eta_{n}, \eta_{n+1}\right)\right)^{a}} \geq S
$$

for all $n \geq n_{0}$. This implies that

$$
n\left(d\left(\eta_{n}, \eta_{n+1}\right)\right)^{a} \leq \operatorname{Rn}\left[\theta\left(d\left(\eta_{n}, \eta_{n+1}\right)\right)-1\right]
$$

for all $n \geq n_{0}$, where $R=\frac{1}{S}$.

Therefore, in all cases, there exist $R>0$ and $n_{0} \in \mathbb{N}$ such that

$$
n\left(d\left(\eta_{n}, \eta_{n+1}\right)\right)^{a} \leq \operatorname{Rn}\left[\theta\left(d\left(\eta_{n}, \eta_{n+1}\right)\right)-1\right],
$$

for all $n \geq n_{0}$. Using (2.4), we can write

$$
n\left(d\left(\eta_{n}, \eta_{n+1}\right)\right)^{a} \leq \operatorname{Rn}\left(\left[\theta\left(d\left(\eta_{0}, \eta_{1}\right)\right)\right]^{\lambda^{n}}-1\right),
$$

for all $n \geq n_{0}$. Letting $n \rightarrow \infty$ in (2.7) we get

$$
\lim _{n \rightarrow \infty} n\left(d\left(\eta_{n}, \eta_{n+1}\right)\right)^{a}=0 .
$$

Hence, there exist $n_{1} \in \mathbb{N}$ such that

$$
d\left(\eta_{n}, \eta_{n+1}\right) \leq \frac{1}{n^{\frac{1}{a}}}, \text { for all } n \geq n_{1} .
$$

For what follows, we shall prove that $\left\{\eta_{n}\right\}$ is a Cauchy sequence by employing standard tools, For any $n, m \in \mathbb{N}$ with $m>n \geq n_{0}$ we obtain

$$
\begin{aligned}
d\left(\eta_{n}, \eta_{m}\right) & \leq d\left(\eta_{n}, \eta_{n+1}\right)+d\left(\eta_{n+1}, \eta_{n+2}\right)+\cdots+d\left(\eta_{m-1}, \eta_{m}\right) \\
& \leq \sum_{i=n}^{m-1} \frac{1}{i^{\frac{1}{a}}} .
\end{aligned}
$$


Since the last term of the above inequality tends to zero as $n, m \rightarrow \infty$, we have $d\left(\eta_{n}, \eta_{m}\right) \rightarrow 0$. As $(X, d)$ is a complete metric spaces, the sequence $\left\{\eta_{n}\right\}$ converges to some point $u \in X$, that is,

$$
\lim _{n \rightarrow \infty} \eta_{n}=u
$$

As a next step make evident that the limit $\eta$ of the iterative sequence $\left\{\eta_{n}\right\}$ is a fixed point of the given mapping $F$. Suppose that $\eta \neq F \eta$, then $d(\eta, F \eta)>0$. By letting $\eta=\eta_{n}$ and $\omega=\eta$ in (2.1), we obtain that

$$
d\left(\eta_{n+1}, F \eta\right)=d\left(F \eta_{n}, F \eta\right) \leq\left[\theta\left(d\left(\eta_{n}, F \eta_{n}\right)\right)\right]^{\lambda \alpha}[\theta(d(\eta, F \eta))]^{\lambda(1-\alpha)} .
$$

Letting $n \rightarrow \infty$ in the above inequality, we obtain, $\eta=F \eta$. Thus the proof is completed.

Remark 2.3. Taking $\theta(t)=e^{t}$ in inequality (2.1), then it turns to Kannan contraction mapping with $\lambda \alpha \in\left[0, \frac{1}{2}\right)$ and $\lambda(1-\alpha) \in\left[0, \frac{1}{2}\right)$.

Definition 2.4. Let $(X, d)$ be a complete metric space and $\theta \in \Theta$. A mapping $F: X \rightarrow K(X)$ is said to be an interpolative multi-valued Kannan type $\theta$-contractive mapping if $\theta \in \Theta$ and there exist $\lambda \in[0,1), \alpha \in(0,1)$ such that

$$
\theta(H(F \eta, F \omega)) \leq[\theta(D(\eta, F \eta))]^{\lambda \alpha}[\theta(D(\omega, F \omega))]^{\lambda(1-\alpha)}
$$

for all $\eta, \omega \in X$.

Theorem 2.5. Let $(X, d)$ be a complete metric space and $F: X \rightarrow K(X)$ be an interpolative multi-valued Kannan type $\theta$-contractive, then $F$ has a fixed point in $X$.

Proof. Let $\eta_{0}$ be an arbitrary point of $X$ and choose a $\eta_{1} \in X$ such that $\eta_{1} \in F \eta_{0}$. Suppose that $\eta_{1} \in F \eta_{1}$, that is, $\eta_{1}$ is a fixed point of $F$. Then, let $\eta_{1} \notin F \eta_{1}$. Since $F \eta_{1}$ is closed, we have $D\left(\eta_{1}, F \eta_{1}\right)>0$ for all $\eta \in X$. On the other hand, from

$$
0<D\left(\eta_{1}, F \eta_{1}\right) \leq H\left(F \eta_{0}, F \eta_{1}\right)
$$

so, from (2.10), and considering $\left(\Theta_{1}\right)$,

$$
\theta\left(D\left(\eta_{1}, F \eta_{1}\right)\right) \leq \theta\left(H\left(F \eta_{0}, F \eta_{1}\right)\right) \leq\left[\theta\left(D\left(\eta_{0}, F \eta_{0}\right)\right)\right]^{\lambda \alpha}\left[\theta\left(D\left(\eta_{1}, F \eta_{1}\right)\right)\right]^{\lambda(1-\alpha)} .
$$

Since $F \eta_{1}$ is compact, there exist $\eta_{2} \in F \eta_{1}$ such that $d\left(\eta_{0}, \eta_{1}\right)=D\left(\eta_{0}, F \eta_{0}\right)$ and $d\left(\eta_{1}, \eta_{2}\right)=D\left(\eta_{1}, F \eta_{1}\right)$. From (2.11), we get

$$
\theta\left(d\left(\eta_{1}, \eta_{2}\right)\right) \leq \theta\left(H\left(F \eta_{0}, F \eta_{1}\right)\right) \leq\left[\theta\left(d\left(\eta_{0}, \eta_{1}\right)\right)\right]^{\lambda \alpha}\left[\theta\left(d\left(\eta_{1}, \eta_{2}\right)\right)\right]^{\lambda(1-\alpha)}
$$

Therefore, continuing recursively, we get $\eta_{n} \in X$ such that $\eta_{n} \in F \eta_{n-1}, \eta_{n+1} \in F \eta_{n}$ and

$$
\theta\left(d\left(\eta_{n}, \eta_{n+1}\right)\right) \leq\left[\theta\left(d\left(\eta_{n-1}, \eta_{n}\right)\right)\right]^{\lambda \alpha}\left[\theta\left(d\left(\eta_{n}, \eta_{n+1}\right)\right)\right]^{\lambda(1-\alpha)} .
$$

If

$$
d\left(\eta_{n-1}, \eta_{n}\right)<d\left(\eta_{n}, \eta_{n+1}\right)
$$

then, from (2.13) we obtain

$$
\theta\left(d\left(\eta_{n}, \eta_{n+1}\right)\right) \leq\left[\theta\left(d\left(\eta_{n}, \eta_{n+1}\right)\right)\right]^{\lambda(1-\alpha+\alpha)}=\left[\theta\left(d\left(\eta_{n}, \eta_{n+1}\right)\right)\right]^{\lambda}
$$

which is a contradiction. Thus, for all $n \in \mathbb{N}$

$$
\theta\left(d\left(\eta_{n}, \eta_{n+1}\right)\right) \leq\left[\theta\left(d\left(\eta_{n-1}, \eta_{n}\right)\right)\right]^{\lambda} .
$$

Denote

$$
\mu_{n}=d\left(\eta_{n}, \eta_{n+1}\right),
$$


for all $n \in \mathbb{N}$. Then, $\mu_{n}>0$ and using (2.14) we have

$$
\theta\left(\mu_{n}\right) \leq\left[\theta\left(\mu_{n-1}\right)\right]^{\lambda} \leq\left[\theta\left(\mu_{n-2}\right)\right]^{\lambda^{2}} \leq \cdots \leq\left[\theta\left(\mu_{0}\right)\right]^{\lambda^{n}} .
$$

Letting $n \rightarrow \infty$ in (2.15) we obtain

$$
\lim _{n \rightarrow \infty} \theta\left(\mu_{n}\right)=1,
$$

From $\left(\Theta_{2}\right)$ we get

$$
\lim _{n \rightarrow \infty} \mu_{n}=0^{+},
$$

and so from $\left(\Theta_{3}\right)$, there exist $a \in(0,1)$ and $b \in(0, \infty]$ such that

$$
\lim _{n \rightarrow \infty} \frac{\theta\left(\mu_{n}\right)-1}{\left(\mu_{n}\right)^{a}}=b
$$

Assume that $b<\infty$. In this case, let $S=\frac{b}{2}>0$. From the definition of the limit, there exist $n_{0} \in \mathbb{N}$ such that

$$
\left|\frac{\theta\left(\mu_{n}\right)-1}{\left(\mu_{n}\right)^{a}}-b\right| \leq S, \text { for all } n \geq n_{0} .
$$

This implies that

$$
\frac{\theta\left(\mu_{n}\right)-1}{\left(\mu_{n}\right)^{a}} \geq b-S=S, \text { for all } n \geq n_{0} .
$$

Thus

$$
n\left(\mu_{n}\right)^{a} \leq R n\left[\theta\left(\mu_{n}\right)-1\right],
$$

for all $n \geq n_{0}$ where $R=\frac{1}{S}$. Now assume that $b=\infty$ and $S>0$ be an arbitrary positive number. From the definition the limit, there exist $n_{0} \in \mathbb{N}$ such that

$$
\frac{\theta\left(\mu_{n}\right)-1}{\left(\mu_{n}\right)^{a}} \geq S
$$

for all $n \geq n_{0}$. This implies that

$$
n\left(\mu_{n}\right)^{a} \leq R n\left[\theta\left(\mu_{n}\right)-1\right],
$$

for all $n \geq n_{0}$, where $R=\frac{1}{S}$.

Therefore, in all cases, there exist $R>0$ and $n_{0} \in \mathbb{N}$ such that

$$
n\left(\mu_{n}\right)^{a} \leq \operatorname{Rn}\left[\theta\left(\mu_{n}\right)-1\right],
$$

for all $n \geq n_{0}$. Using (2.15), we obtain

$$
n\left(\mu_{n}\right)^{a} \leq R n\left(\left[\theta\left(\mu_{0}\right)\right]^{\lambda^{n}}-1\right),
$$

for all $n \geq n_{0}$. Letting $n \rightarrow \infty$ in (2.18) we get

$$
\lim _{n \rightarrow \infty} n\left(\mu_{n}\right)^{a}=0 .
$$

Therefore, there exist $n_{1} \in \mathbb{N}$ such that

$$
\mu_{n} \leq \frac{1}{n^{\frac{1}{a}}}, \text { for all } n \geq n_{1} .
$$


For what follows, we shall prove that $\left\{\eta_{n}\right\}$ is a Cauchy sequence by employing standard tools, For any $n, m \in \mathbb{N}$ with $m>n \geq n_{0}$ we obtain

$$
\begin{aligned}
d\left(\eta_{n}, \eta_{m}\right) & \leq d\left(\eta_{n}, \eta_{n+1}\right)+d\left(\eta_{n+1}, \eta_{n+2}\right)+\cdots+d\left(\eta_{m-1}, \eta_{m}\right) \\
& =\mu_{n}+\mu_{n+1}+\cdots+\mu_{m-1} \\
& \leq \sum_{i=n}^{m-1} \frac{1}{i^{\frac{1}{a}}} .
\end{aligned}
$$

Since the last term of the above inequality tends to zero as $n, m \rightarrow \infty$, we have $d\left(\eta_{n}, \eta_{m}\right) \rightarrow 0$. As $(X, d)$ is a complete metric spaces, the sequence $\left\{\eta_{n}\right\}$ converges to some point $u \in X$, that is,

$$
\lim _{n \rightarrow \infty} \eta_{n}=u \text {. }
$$

Case 1: There is a subsequence $\left\{\eta_{n_{r}}\right\}$ such that $F \eta_{n_{r}}=F u$ for all $r \in \mathbb{N}$. In this case,

$$
D(u, F u)=\lim _{n \rightarrow \infty} D\left(\eta_{n_{r+1}}, F u\right) \leq \lim _{n \rightarrow \infty} H\left(F \eta_{n_{r}}, F u\right)=0 .
$$

Case 2: There is a natural number $N$ such that $F \eta_{n} \neq F u$ for all $n \geq N$. In this cases applying (2.10) for $u=\eta_{n}$ and $\omega=u$ we have

$$
\theta\left(D\left(\eta_{n+1}, F u\right)\right) \leq \theta\left(H\left(F \eta_{n}, F u\right)\right) \leq\left[\theta\left(D\left(\eta_{n}, F \eta_{n}\right)\right)\right]^{\lambda \alpha}[\theta(D(u, F u))]^{\lambda(1-\alpha)} .
$$

Then assume that

$$
D\left(\eta_{n}, F \eta_{n}\right)<D(u, F u),
$$

letting $n \rightarrow \infty$ in (2.21) we obtain,

$$
\theta(D(u, F u)) \leq[\theta(D(u, F u))]^{\lambda}
$$

which is a contradiction. Then we obtain

$$
D(u, F u) \leq D\left(\eta_{n}, F \eta_{n}\right),
$$

so, we get

$$
\theta(D(u, F u)) \leq\left[\theta\left(D\left(\eta_{n}, F \eta_{n}\right)\right)\right]^{\lambda} .
$$

Since $F \eta_{n}$ is compact, there exist $\eta_{n+1} \in F \eta_{n}$ such that $d\left(\eta_{n}, \eta_{n+1}\right)=D\left(\eta_{n}, F \eta_{n}\right)$. Since (2.22), we get

$$
\theta(D(u, F u)) \leq\left[\theta\left(D\left(\eta_{n}, \eta_{n+1}\right)\right)\right]^{\lambda}
$$

letting $n \rightarrow \infty$ in (2.23) we obtain, $u \in F u$. Thus the proof is completed.

Hançer et al. [15], showed that we can take " $C B(X)$ " instead of " $K(X)$ ", by adding the condition $\left(\theta_{4}\right)$ on $\theta:(0, \infty) \rightarrow(1, \infty)$, as follows:

$\left(\theta_{4}\right) \theta(\inf M)=\inf \theta(M)$ for all $M \subset(0, \infty)$ with $\inf M>0$.

Take in the consideration if $\theta$ is right continuous and satisfies $\left(\theta_{1}\right)$, in that case $\left(\theta_{4}\right)$ founds. Let $\Xi$ be the family of all functions $\theta$ satisfying $\left(\theta_{1}\right)-\left(\theta_{4}\right)$.

Corollary 2.6. Let $(X, d)$ be a complete metric space and $F: X \rightarrow C B(X)$ be a mapping. Given that there are $\theta \in \Xi, \lambda \in[0,1)$ and $\alpha \in(0,1)$ such that

$$
\theta(H(F \eta, F \omega)) \leq[\theta(D(\eta, F \eta))]^{\lambda \alpha}[\theta(D(\omega, F \omega))]^{\lambda(1-\alpha)}
$$

for all $\eta, \omega \in X$ then $F$ has a fixed point in $X$. 
Proof. Let $\eta_{0}$ be an arbitrary point of $X$ and choose a $\eta_{1} \in X$ such that $\eta_{1} \in F \eta_{0}$. Assume that $\eta_{1} \in F \eta_{1}$, that is, $\eta_{1}$ is a fixed point of $F$. Then, let $\eta_{1} \notin F \eta_{1}$. As $F \eta_{1}$ is closed, we obtain $D\left(\eta_{1}, F \eta_{1}\right)>0$ for all $\eta \in X$. So, from (2.24), and considering $\left(\Theta_{1}\right)$,

$$
\theta\left(D\left(\eta_{1}, F \eta_{1}\right)\right) \leq \theta\left(H\left(F \eta_{0}, F \eta_{1}\right)\right) \leq\left[\theta\left(D\left(\eta_{0}, F \eta_{0}\right)\right)\right]^{\lambda \alpha}\left[\theta\left(D\left(\eta_{1}, F \eta_{1}\right)\right)\right]^{\lambda(1-\alpha)} .
$$

Considering condition $\left(\theta_{4}\right)$, we obtain $\theta\left(D\left(\eta_{1}, F \eta_{1}\right)\right)=\inf _{z \in F \eta_{1}} \theta\left(d\left(\eta_{1}, z\right)\right)$. Then we have

$$
\begin{aligned}
\inf _{z \in F \eta_{1}} \theta\left(d\left(\eta_{1}, z\right)\right) & \leq\left[\theta\left(D\left(\eta_{0}, F \eta_{0}\right)\right)\right]^{\lambda \alpha}\left[\theta\left(D\left(\eta_{1}, F \eta_{1}\right)\right)\right]^{\lambda(1-\alpha)} \\
& <\left[\theta\left(D\left(\eta_{0}, F \eta_{0}\right)\right)\right]^{\lambda_{1} \alpha}\left[\theta\left(D\left(\eta_{1}, F \eta_{1}\right)\right)\right]^{\lambda_{1}(1-\alpha)}
\end{aligned}
$$

where $\lambda_{1} \in(\lambda, 1)$. Then, from (2.26), there exist $\eta_{1} \in F \eta_{0}$ and $\eta_{2} \in F \eta_{1}$ such that

$$
\theta\left(d\left(\eta_{1}, \eta_{2}\right)\right) \leq\left[\theta\left(d\left(\eta_{0}, \eta_{1}\right)\right)\right]^{\lambda_{1} \alpha}\left[\theta\left(d\left(\eta_{1}, \eta_{2}\right)\right)\right]^{\lambda_{1}(1-\alpha)} .
$$

The rest of the proof can be completed as in the proof of Theorem 2.5.

Definition 2.7. Let $(X, d)$ be a complete metric space and $\theta \in \Theta$. A mapping $F: X \rightarrow X$ is said to be an interpolative Reich Rus Ćirić type $\theta$-contractive mapping if $\theta \in \Theta$ and there exist $\lambda \in[0,1), \beta, \alpha \in(0,1)$ with $\beta+\alpha<1$ such that

$$
\theta(d(F \eta, F \omega)) \leq[\theta(d(\eta, \omega))]^{\lambda \beta}[\theta(d(\eta, F \eta))]^{\lambda \alpha}[\theta(d(\omega, F \omega))]^{\lambda(1-\beta-\alpha)}
$$

for all $\eta, \omega \in X$.

Theorem 2.8. Let $(X, d)$ be a complete metric space and $F: X \rightarrow X$ be an interpolative Reich Rus Cirić type $\theta$-contractive, then $F$ has a fixed point in $X$.

Proof. Starting from $\eta_{0} \in X$, consider $\left\{\eta_{n}\right\}$ given as $\eta_{n}=F \eta_{n-1}$ for all positive integer $n$. If there is $n_{0}$ so that $\eta_{n 0}=\eta_{n 0+1}$ then $\eta_{n 0}$ is a fixed point of $F$. Assume that $\eta_{n} \neq \eta_{n+1}$ for all $n \geq 0$. Taking $\eta=\eta_{n-1}$ and $\omega=\eta_{n}$ in (2.28), one writes

$$
\theta\left(d\left(\eta_{n}, \eta_{n+1}\right)\right) \leq\left[\theta\left(d\left(\eta_{n-1}, \eta_{n}\right)\right)\right]^{\lambda \beta}\left[\theta\left(d\left(\eta_{n-1}, \eta_{n}\right)\right)\right]^{\lambda \alpha}\left[\theta\left(d\left(\eta_{n}, \eta_{n+1}\right)\right)\right]^{\lambda(1-\beta-\alpha)} .
$$

If

$$
d\left(\eta_{n-1}, \eta_{n}\right)<d\left(\eta_{n}, \eta_{n+1}\right)
$$

then, from (2.29) we obtain

$$
\theta\left(d\left(\eta_{n}, \eta_{n+1}\right)\right) \leq\left[\theta\left(d\left(\eta_{n}, \eta_{n+1}\right)\right)\right]^{\lambda(\beta+\alpha+1-\alpha-\beta)}=\left[\theta\left(d\left(\eta_{n}, \eta_{n+1}\right)\right)\right]^{\lambda}
$$

which is a contradiction. Thus, for all $n \in \mathbb{N}$

$$
\theta\left(d\left(\eta_{n}, \eta_{n+1}\right)\right) \leq\left[\theta\left(d\left(\eta_{n-1}, \eta_{n}\right)\right)\right]^{\lambda} .
$$

From (2.30) we have

$$
\theta\left(d\left(\eta_{n}, \eta_{n+1}\right)\right) \leq\left[\theta\left(d\left(\eta_{n-1}, \eta_{n}\right)\right)\right]^{\lambda} \leq\left[\theta\left(d\left(\eta_{n-2}, \eta_{n-1}\right)\right)\right]^{\lambda^{2}} \leq \cdots \leq\left[\theta\left(d\left(\eta_{0}, \eta_{1}\right)\right)\right]^{\lambda^{n}} .
$$

Then, it can be seen that the $\left\{\eta_{n}\right\}$ is a Cauchy with similar operations in Theorem 2.2. As $(X, d)$ is a complete metric spaces, the sequence $\left\{\eta_{n}\right\}$ converges to some point $u \in X$, that is,

$$
\lim _{n \rightarrow \infty} d\left(\eta_{n}, \eta_{n+1}\right)=u .
$$

As a next step make evident that the limit $\eta$ of the iterative sequence $\left\{\eta_{n}\right\}$ is a fixed point of the given mapping $F$. Suppose that $\eta \neq F \eta$, then $d(\eta, F \eta)>0$. By letting $\eta=\eta_{n}$ and $\omega=\eta$ in (2.28), we obtain that

$$
d\left(\eta_{n+1}, F \eta\right)=d\left(F \eta_{n}, F \eta\right) \leq\left[\theta\left(d\left(\eta_{n}, \eta\right)\right)\right]^{\lambda \beta}\left[\theta\left(d\left(\eta_{n}, F \eta_{n}\right)\right)\right]^{\lambda \alpha}[\theta(d(\eta, F \eta))]^{\lambda(1-\alpha-\beta)} .
$$

Letting $n \rightarrow \infty$ in the above inequality, we obtain, $\eta=F \eta$. Thus the proof is completed. 
Remark 2.9. Taking $\theta(t)=e^{t}$ in inequality (2.28), then it turns to Reich-Rus-Ćirić contraction mapping with $a, b, c \in(0,1)$ such that $\lambda \beta=a, \lambda \alpha=b$ and $c=1-\beta-\alpha, 0 \leq a+b+c<1$.

Definition 2.10. Let $(X, d)$ be a complete metric space and $\theta \in \Theta$. A mapping $F: X \rightarrow K(X)$ is said to be an interpolative multi-valued Reich Rus Ćirić type $\theta$-contractive mapping if $\theta \in \Theta$ and there exist $\lambda \in[0,1), \beta, \alpha \in(0,1)$ with $\beta+\alpha<1$ such that

$$
\theta(H(F \eta, F \omega)) \leq[\theta(d(\eta, \omega))]^{\lambda \beta}[\theta(D(\eta, F \eta))]^{\lambda \alpha}[\theta(D(\omega, F \omega))]^{\lambda(1-\beta-\alpha)}
$$

for all $\eta, \omega \in X$.

Theorem 2.11. Let $(X, d)$ be a complete metric space and $F: X \rightarrow K(X)$ be an interpolative multi-valued Reich Rus Cirić type $\theta$-contractive, then $F$ has a fixed point in $X$.

Proof. Let $\eta_{0} \in X$. Since $F \eta$ is nonempty for all $\eta_{0} \in X$, we can chose a $\eta_{1} \in X$. Assume that $\eta_{1} \in F \eta_{1}$, that is, $\eta_{1}$ is a fixed point of $F$. Now, let $\eta_{1} \notin F \eta_{1}$. As $F \eta_{1}$ is closed, we obtain $D\left(\eta_{1}, F \eta_{1}\right)>0$ for all $\eta \in X$. Moreover, as

$$
0<d\left(\eta_{1}, F \eta_{1}\right) \leq H\left(F \eta_{0}, F \eta_{1}\right),
$$

from (2.33) and considering $\left(\Theta_{1}\right)$, we can write that

$$
\begin{aligned}
\theta\left(d\left(\eta_{1}, F \eta_{1}\right)\right) & \leq \theta\left(H\left(F \eta_{0}, F \eta_{1}\right)\right) \\
& \leq\left[\theta\left(d\left(\eta_{0}, \eta_{1}\right)\right)\right]^{\lambda \beta}\left[\theta\left(d\left(\eta_{0}, F \eta_{0}\right)\right)\right]^{\lambda \alpha}\left[\theta\left(d\left(\eta_{1}, F \eta_{1}\right)\right)\right]^{\lambda(1-\beta-\alpha)} .
\end{aligned}
$$

As $F \eta_{1}$ is compact, there exist $\eta_{2} \in F \eta_{1}$ such that $d\left(\eta_{0}, \eta_{1}\right)=d\left(\eta_{0}, F \eta_{0}\right)$ and $d\left(\eta_{1}, \eta_{2}\right)=d\left(\eta_{1}, F \eta_{1}\right)$. From (2.34), we obtain

$$
\begin{aligned}
\theta\left(d\left(\eta_{1}, \eta_{2}\right)\right) & \leq \theta\left(H\left(F \eta_{0}, F \eta_{1}\right)\right) \\
& \leq\left[\theta\left(d\left(\eta_{0}, \eta_{1}\right)\right)\right]^{\lambda \beta}\left[\theta\left(d\left(\eta_{0}, \eta_{1}\right)\right)\right]^{\lambda \alpha}\left[\theta\left(d\left(\eta_{1}, \eta_{2}\right)\right)\right]^{\lambda(1-\beta-\alpha)} .
\end{aligned}
$$

Therefore, continue recursively, we get $\eta_{n} \in X$ such that $\eta_{n} \in F \eta_{n-1}, \eta_{n+1} \in F \eta_{n}$, and

$$
\theta\left(d\left(\eta_{n}, \eta_{n+1}\right)\right) \leq\left[\theta\left(d\left(\eta_{n-1}, \eta_{n}\right)\right)\right]^{\lambda \beta}\left[\theta\left(d\left(\eta_{n-1}, \eta_{n}\right)\right)\right]^{\lambda \alpha}\left[\theta\left(d\left(\eta_{n}, \eta_{n+1}\right)\right)\right]^{\lambda(1-\beta-\alpha)} .
$$

Suppose that

$$
d\left(\eta_{n-1}, \eta_{n}\right)<d\left(\eta_{n}, \eta_{n+1}\right),
$$

then from (2.36) we obtain

$$
\theta\left(d\left(\eta_{n}, \eta_{n+1}\right)\right) \leq\left[\theta\left(d\left(\eta_{n}, \eta_{n+1}\right)\right)\right]^{\lambda(\beta+\alpha+1-\alpha-\alpha)}=\left[\theta\left(d\left(\eta_{n}, \eta_{n+1}\right)\right)\right]^{\lambda}
$$

which is a contradiction. Therefore for all $n \in \mathbb{N}$

$$
\theta\left(d\left(\eta_{n}, \eta_{n+1}\right)\right) \leq\left[\theta\left(d\left(\eta_{n-1}, \eta_{n}\right)\right)\right]^{\lambda} .
$$

Let

$$
\mu_{n}=d\left(\eta_{n}, \eta_{n+1}\right),
$$

for all $n \in \mathbb{N}$. Thus, $\mu_{n}>0$ and handling (2.37) we get

$$
\theta\left(\mu_{n}\right) \leq\left[\theta\left(\mu_{n-1}\right)\right]^{\lambda} \leq\left[\theta\left(\mu_{n-2}\right)\right]^{\lambda^{2}} \leq \cdots \leq\left[\theta\left(\mu_{0}\right)\right]^{\lambda^{n}} .
$$

Then, it can be seen that the $\left\{\eta_{n}\right\}$ is a Cauchy with similar operations in Theorem 2.5.

Since $(X, d)$ is a complete metric spaces, the sequence $\left\{\eta_{n}\right\}$ converges to some point $u \in X$, that is,

$$
\lim _{n \rightarrow \infty} \eta_{n}=u .
$$

Case 1: There is a subsequence $\left\{\eta_{n_{r}}\right\}$ such that $F \eta_{n_{r}}=F u$ for all $r \in \mathbb{N}$. Therefore,

$$
D(u, F u)=\lim _{n \rightarrow \infty} D\left(\eta_{n_{r+1}}, F u\right) \leq \lim _{n \rightarrow \infty} H\left(F \eta_{n_{r}}, F u\right)=0 .
$$


Case 2: There is a natural number $N$ such that $F \eta_{n} \neq F u$ for all $n \geq N$. In this cases applying (2.33) for $u=\eta_{n}$ and $\omega=u$ we have

$$
\begin{aligned}
\theta\left(D\left(\eta_{n+1}, F u\right)\right) & \leq \theta\left(H\left(F \eta_{n}, F u\right)\right) \\
& \leq\left[\theta\left(D\left(\eta_{n}, u\right)\right)\right]^{\lambda \beta}\left[\theta\left(D\left(\eta_{n}, F \eta_{n}\right)\right)\right]^{\lambda \alpha}[\theta(D(u, F u))]^{\lambda(1-\beta-\alpha)} .
\end{aligned}
$$

Hence, suppose that

$$
D\left(\eta_{n}, F \eta_{n}\right)<D(u, F u)
$$

letting $n \rightarrow \infty$ in (2.40) we get,

$$
\theta(D(u, F u)) \leq[\theta(D(u, F u))]^{\lambda(1-\beta)}
$$

which is a contradiction. So we obtain

$$
D(u, F u) \leq D\left(\eta_{n}, F \eta_{n}\right),
$$

then, we get

$$
\theta(D(u, F u)) \leq\left[\theta\left(D\left(\eta_{n}, F \eta_{n}\right)\right)\right]^{\lambda(1-\beta)} .
$$

As $F \eta_{n}$ is compact, there exist $\eta_{n+1} \in F \eta_{n}$ such that $d\left(\eta_{n}, \eta_{n+1}\right)=D\left(\eta_{n}, F \eta_{n}\right)$. From (2.41), we obtain

$$
\theta(D(u, F u)) \leq\left[\theta\left(D\left(\eta_{n}, \eta_{n+1}\right)\right)\right]^{\lambda(1-\beta)}
$$

letting $n \rightarrow \infty$ in (2.42) we get, $u \in F u$. Therefore the proof is completed.

Example 2.12. Let $X=[0, \infty)$ and define $d(\eta, \omega)=|\eta-\omega|$, for all $\eta, \omega \in X .(X, d)$ is a complete metric space. Also defined $F: X \rightarrow K(X)$ a mapping, where

$$
F \eta= \begin{cases}\{0\}, & \text { if } \eta \in[0,1) \\ \left\{\frac{\eta}{6}\right\}, & \text { if } \eta \in[1, \infty)\end{cases}
$$

Let $\lambda=\frac{1}{\sqrt{2}}, \beta=\frac{1}{2}, \alpha=\frac{1}{3}$ and $\theta(m)=e^{m}$ pertain to $\Theta$. Without loss of generality, we may assume that $\eta \geq \omega$. Thus, through a series of standard calculations, we can proved that

$$
\theta(H(F \eta, F \omega)) \leq[\theta(D(\eta, \omega))]^{\lambda \beta}[\theta(D(\eta, F \eta))]^{\lambda \alpha}[\theta(D(\omega, F \omega))]^{\lambda(1-\beta-\alpha)}
$$

for all $\eta, \omega \in X$. So, this is satisfying the condition of Theorem 2.11. F has fixed points. Since similar process are performed, the condition of Theorem 2.5 is satisfied.

Corollary 2.13. Let $(X, d)$ be a complete metric space and $F: X \rightarrow C B(X)$ be a mapping. Suppose that there are $\theta \in \Xi$, $\lambda \in[0,1)$ and $\beta, \alpha \in(0,1)$ with $\beta+\alpha<1$ such that

$$
\theta(H(F \eta, F \omega)) \leq[\theta(d(\eta, \omega))]^{\lambda \beta}[\theta(d(\eta, F \eta))]^{\lambda \alpha}[\theta(d(\omega, F \omega))]^{\lambda(1-\beta-\alpha)}
$$

for all $\eta, \omega \in X$ then $F$ has a fixed point in $X$.

Proof. Let $\eta_{0}$ be an arbitrary point of $X$ and choose a $\eta_{1} \in X$ such that $\eta_{1} \in F \eta_{0}$. Assume that $\eta_{1} \in F \eta_{1}$, that is, $\eta_{1}$ is a fixed point of $F$. Therefore, let $\eta_{1} \notin F \eta_{1}$. Since $F \eta_{1}$ is closed, we obtain $D\left(\eta_{1}, F \eta_{1}\right)>0$ for all $\eta \in X$. Hence, from (2.43), and considering $\left(\Theta_{1}\right)$, we can write

$$
\begin{aligned}
\theta\left(D\left(\eta_{1}, F \eta_{1}\right)\right) & \leq \theta\left(H\left(F \eta_{0}, F \eta_{1}\right)\right) \\
& \leq\left[\theta\left(d\left(\eta_{0}, \eta_{1}\right)\right)\right]^{\lambda \beta}\left[\theta\left(d\left(\eta_{0}, F \eta_{0}\right)\right)\right]^{\lambda \alpha}\left[\theta\left(d\left(\eta_{1}, F \eta_{1}\right)\right)\right]^{\lambda(1-\beta-\alpha)} .
\end{aligned}
$$

Considering condition $\left(\theta_{4}\right)$, we get $\theta\left(D\left(\eta_{1}, F \eta_{1}\right)\right)=\inf _{z \in F \eta_{1}} \theta\left(d\left(\eta_{1}, z\right)\right)$. Thus, we have

$$
\begin{aligned}
\inf _{z \in F \eta_{1}} \theta\left(d\left(\eta_{1}, z\right)\right) & \leq\left[\theta\left(d\left(\eta_{0}, \eta_{1}\right)\right)\right]^{\lambda \beta}\left[\theta\left(d\left(\eta_{0}, F \eta_{0}\right)\right)\right]^{\lambda \alpha}\left[\theta\left(d\left(\eta_{1}, F \eta_{1}\right)\right)\right]^{\lambda(1-\beta-\alpha)} \\
& <\left[\theta\left(d\left(\eta_{0}, \eta_{1}\right)\right)\right]^{\lambda_{1} \beta}\left[\theta\left(d\left(\eta_{0}, F \eta_{0}\right)\right)\right]^{\lambda_{1} \alpha}\left[\theta\left(d\left(\eta_{1}, F \eta_{1}\right)\right)\right]^{\lambda_{1}(1-\beta-\alpha)}
\end{aligned}
$$

where $\lambda_{1} \in(\lambda, 1)$. Then, from (2.45), there exist $\eta_{1} \in F \eta_{0}$ and $\eta_{2} \in F \eta_{1}$ such that

$$
\theta\left(d\left(\eta_{1}, \eta_{2}\right)\right) \leq\left[\theta\left(d\left(\eta_{0}, \eta_{1}\right)\right)\right]^{\lambda_{1} \beta}\left[\theta\left(d\left(\eta_{0}, \eta_{1}\right)\right)\right]^{\lambda_{1} \alpha}\left[\theta\left(d\left(\eta_{1}, \eta_{2}\right)\right)\right]^{\lambda_{1}(1-\beta-\alpha)} .
$$

The rest of the proof can be completed as in the proof of Theorem 2.11. 


\section{Conclusion}

We aimed to present new some results to the fixed point theory by combining the ideas of Nadler, Karapınar et. al., Jleli and Samet. We introduce the concept of interpolative single and multi-valued Kannan type and Reich Rus Ćirić type $\theta$-contractive mappings metric spaces and prove some fixed point results for such mappings.

\section{Acknowledgement}

The authors wish to thank the referees for their careful reading of the manuscript and valuable suggestions.

\section{References}

[1] S.B. Nadler, Multivalued contraction mappings, Pacific Journal of Mathematics, 30 (1969), 475-488.

[2] E. Karapinar, Revisiting the Kannan type contractions via interpolation, Advances in the Theory of Nonlinear Analysis and its Applications, 2 (2018), 85-87.

[3] E. Karapinar, R.P. Agarwal, H. Aydi, Interpolative Reich-Rus-Ćirić Type Contractions on Partial Metric Spaces, Mathematics , 6(11) (2018), 256.

[4] M. Jleli, B. Samet, A new generalization of the Banach contraction principle, Journal of Inequalities and Applications, 38 (2014), 1-8.

[5] S. Banach, Sur les operations dans les ensembles abstracits et leur application aux equations integrales, Fund. Math., 3 (1922), 133-181.

[6] R. Kannan, Some results on fixed points, Bull. Cal. Math. Soc., 60 (1968), 71-76.

[7] S. Reich, Fixed point of contractive functions, Boll. Unione Mat. Ital., 5 (1972), 26-42.

[8] L. B. Ćirić, Generalized contractions and fixed point theorems, Publ. Inst. Math. (Beograd)(NS), 12 (1971), $19-26$.

[9] S. Reich, Some remarks concerning contraction mappings, Canadian Mathematical Bulletin, 14 (1971), $121-124$.

[10] L.B, Ćirić, On contraction type mappings, Math. Balk., 1 (1971), 52-57.

[11] L.B, Ćirić, Generalized contractions and fixed point theorems, Publ. Inst. Math. (Belgr.), 12 (1971), 19-26.

[12] S. Reich, Kannan's fixed point theorem, Boll. Unione Mat. Ital., 4 (1971), 1-11.

[13] I.A. Rus, Principles and applications of the fixed point theory, Editura Dacia, Clui-Napoca, Romania, (1979).

[14] I.A. Rus, Generalized contractions and applications; Cluj University Press: Clui-Napoca, Romania, (2001).

[15] H. A. Hançer, G. Mınak, I. Altun, On a broad category of multivalued weakly Picard operators, Fixed point theory, 18 (2017) 229-236. 